\title{
Pactes ruraux post-2014 entre Pays et Parc naturel régional : quels territoires de développement et d'aménagement des campagnes québécoises ?
}

\author{
Louis Allie \\ Université du Québec à Montréal
}

Le Pacte rural, un concept original de développement du milieu rural, est la pierre angulaire de la Politique nationale de la ruralité (PNR) instituée en 2001 et renouvelée de 2007 à 2014. Cet article cherche à mettre en valeur le Pacte rural en réfléchissant sur son avenir dans une perspective comparative, conceptuelle, organisationnelle et territoriale. Il ne s'agit pas de le remettre en cause, car cet outil est fort apprécié sur le terrain et il représente une façon innovatrice et nécessaire de faire du développement rural $^{1}$, bien que l'échelle d'intervention retenue, à savoir celle de la Municipalité régionale de comté (MRC), ne soit pas forcément toujours justifiée. Nous constatons qu'après plus de six années de fonctionnement et de mise en œuvre, il est possible d'affiner l'outil Pacte rural afin de lui permettre de mieux atteindre ses objectifs. En l'état actuel, les concepteurs du Pacte rural (en l'occurrence le ministère des Régions sous le règne péquiste et le ministère des Affaires municipales et des Régions du Parti libéral depuis 2003) ont fait de cet outil avant tout un mode d'intervention étatique de type contractuel au risque de perdre de vue l'hypothèse territoriale qui, pourtant, les a fait naître ${ }^{2}$.

Pour mieux atteindre nos objectifs, nous comparons le Pacte rural avec deux politiques similaires en France que sont les Pays institués en 1995 et les Parcs naturels régionaux décrétés en $1967^{3}$. Avant d'aborder cette approche comparative, nous analysons la portée et la limite du Pacte rural en commençant par leur définition.

\section{Le pacte rural à l'épreuve du territoire}

La raison d'être du Pacte rural est de promouvoir le développement du milieu rural, et en particulier de promouvoir le renouvellement et l'intégration des populations; de favoriser la mise en valeur des ressources humaines, culturelles et physiques du territoire; d'assurer la pérennité des communautés rurales; et enfin, de maintenir un équilibre entre la qualité de vie, le cadre de vie, l'environnement naturel et les activités économiques. Ces objectifs sont à la hauteur des problématiques qui affectent les milieux ruraux. Cependant, en excluant, jusqu'à un certain point, l'enveloppe budgétaire dé-

diée au Pacte rural, l'écart entre la finalité et les moyens avancés (organisationnel et conceptuel) est important.

Un contrat type incarne le Pacte rural qui lie le gouvernement aux MRC. Ce contrat compte treize clauses dont huit expliquent les engagements respectifs de la MRC et de la ministre des Affaires municipales et des régions (MAMR). Les trois signataires du contrat sont la ministre du MAMR, le ministre responsable de la Région et le préfet de la MRC.

Le contrat type repose sur trois engagements majeurs de la ministre. Elle s'engage à réserver une somme de 213 millions de dollars aux MRC et aux municipalités signataires d'un Pacte rural. Elle s'engage deuxièmement à accorder une aide financière pour l'embauche d'un, de deux ou de trois agents de développement (sous la responsabilité du Centre local de développement) pour faciliter la réalisation du Pacte rural. Enfin, la ministre s'engage à ce que la direction générale du MAMR confie à un comité de la Conférence administrative régionale le support à la mise en œuvre de la Politique nationale de la ruralité. Quant à la MRC, elle s'engage à produire un plan de travail à la signature du contrat qu'elle doit actualiser une fois par an- 
née. Les autres clauses sont de nature administrative et légale telle que les recours à envisager en cas de non-respect du contrat.

Le plan de travail «doit s'appuyer sur la notion de territoire » et en particulier « sur une lecture commune de l'état de la situation socioéconomique à l'échelle de la MRC », tout en déterminant les résultats attendus. La MRC doit prévoir les modalités de mobilisation, d'animation, d'information et de reddition de comptes à la population et à la ministre. Elle doit veiller à ce que les activités du Pacte rural répondent au principe de transparence dans l'utilisation des ressources financières, dans le déroulement des projets et dans la diffusion des résultats.

\section{En l'état actuel, la procédure Pacte rural encourage plutôt les acteurs locaux à administrer le territoire par le biais d'une enveloppe budgétaire et indirectement à le développer.}

\section{Des territoires pourtant pluriels}

La formule retenue est relativement simple: plus d'argent pour répondre aux besoins de la population, pour améliorer des équipements et pour organiser des activités, de même que plus d'argent aussi pour monter des projets initiés ou non par la MRC. Le contrat type oublie de préciser que le Pacte rural doit avant tout être un vaste projet territorialisé en soi. Ainsi, les initiatives financées sont souvent davantage cosmétiques que territoriales lorsqu'il y a absence de véritable projet social (ou contrat moral) auquel adhère l'ensemble de la population ${ }^{4}$.

Pour des raisons, notamment de pressions politiques et des relations de travail conventionnées entre les paliers gouvernementaux, l'échelle d'intervention du gouvernement n'a pas été repensée en 2001 au moment où le Pacte rural a été créé. Ainsi, le Pacte rural a été institué pour la MRC (une forme de décentralisation) et il devient par le fait même une extension de l'État à cet échelon : les MRC demeure des organisations fonctionnelles publiques ${ }^{5}$. Toute la réflexion (d'une durée de plus de cinq ans) qui a précédé l'adoption de la première Politique nationale de la ruralité s'est focalisée sur un seul objectif: donner plus de moyens décentralisés (sous-entendu des moyens financiers) aux acteurs locaux du monde ru- ral. Compte tenu de la formulation actuelle du contrat type d'un Pacte rural, la quête du bon périmètre d'intervention est passée sous silence. En considérant $a$ priori acquise la pertinence des MRC comme échelle d'application des Pactes ruraux, le ministère court le risque de renforcer la logique administrative du développement au détriment de la logique territoriale.

L'élaboration d'un Pacte rural pourrait être l'occasion de réviser les affiliations municipales entre les élus, les citoyens et les acteurs locaux au sein d'une ou de plusieurs MRC tout en définissant de nouvelles bases communes de discussions comme, par exemple, dans le cas des MRC des Sources et d'Arthabaska qui, en septembre 2007, se sont associées pour créer une nouvelle structure unique au Québec : la Régie intermunicipale de restauration et de préservation des TroisLacs. Ce type d'organisation est intéressant dans la mesure où il s'appuie sur une volonté sociopolitique locale et une problématique territoriale régionale. Par extension, l'organisme de gestion (qui reste à concevoir) d'un Pacte rural pourrait s'inspirer de cette façon de faire ad hoc sans pour autant promouvoir cette seule vocation. Le Pacte rural devrait normalement représenter une occasion aussi et surtout de discuter du territoire, de ses caractéristiques, de ses difficultés et de son avenir. En l'état actuel, la procédure Pacte rural encourage plutôt les acteurs locaux à administrer le territoire par le biais d'une enveloppe budgétaire et indirectement à le développer : le ministère concourt à promouvoir une logique comptable et non une vision territoriale. Ainsi, le contrat type du Pacte rural envoie le signal selon lequel les élus des MRC administrent une enveloppe budgétaire sans qu'ils soient réellement contraints dans l'affectation des ressources financières à l'intérieur du large cadre prévu par la Politique nationale de la ruralité.

\section{Le pacte rural : un contrat territorial sans politique de territorialisation}

En choisissant d'emblée la MRC et cette échelle d'intervention comme lieu de mise en œuvre du Pacte rural, l'État n'a-t-il pas raté l'occasion d'ajuster ses interventions au profit des acteurs de terrain pour mieux appréhender le territoire dans toute sa complexité et ses dimensions (sociopolitique, culturelle, économique et géographique) ? La montée en puissance des Pactes ruraux ne serait-elle pas une occasion d'ajuster l'échelle d'intervention de l'État aux besoins des communautés rurales? 
La Loi sur l'aménagement et l'urbanisme (ou loi 125), sanctionnée le 21 novembre 1979, a institué les MRC (au nombre de 86 auxquels s'ajoutent 14 villes et agglomération exerçant certaines compétences de MRC). En vertu des lois en vigueur (LRQ, chapitre A-19.1 et chapitre C-47-1 a.104-110), la MRC assume obligatoirement les responsabilités suivantes: maintenir en vigueur un schéma d'aménagement et de développement; voir à ce que les plans et les règlements d'urbanisme des municipalités soient conformes au schéma d'aménagement; désigner ou consolider un Centre local de développement en plus de nombreuses compétences déléguées qui se sont multipliées depuis le début 2000.

La délimitation de leur périmètre ne s'est pas faite sans heurts, et cet exercice répondait à des logiques différentes selon le contexte local et régional du moment ${ }^{6}$.

Certaines MRC ont été délimitées en incluant d'emblée des villes de petite et moyenne taille (c'est le cas de la MRC de Joliette), alors que dans d'autres cas ce fut l'inverse (par exemple, le cas des MRC Memprémagog, Le Val-Saint-François, Le Haut-Saint-François et Coaticook définies en opposition à l'agglomération de Sherbrooke). Dans ce cas de figure, la proximité urbaine était ou bien considérée comme un atout ou bien comme une nuisance. Les oppositions ville/ campagne - aussi vieilles que le système municipal québécois - se matérialisent en particulier par des prises de position opposées entre la Fédération québécoise des municipalités (FQM) et l'Union des municipalités du Québec (UMQ). En octobre 2007, la ministre des Affaires municipales et des Régions, Nathalie Normandeau, qui affirmait vouloir favoriser les solidarités entre les milieux rural et urbain, semble avoir provoqué l'inverse : le ministère a accordé une subvention de $450000 \$$ à l'UMQ pour soutenir une réflexion sur les partenariats urbains-ruraux. Pour la FQM, ce n'est pas à la « ville » de penser l'avenir du milieu rural; cette union croit plutôt a contrario que le «monde rural» est le plus apte à penser l'avenir des villes.

Dans cet effort de découpage des MRC, le ministère a parfois entériné des périmètres proposés par les élus locaux qui correspondent souvent à des liens personnels et relationnels (entre les maires ruraux des MRC de Rouville et des Jardins-de-Napierville, par exemple); alors que, dans d'autres cas, il a plutôt obligé la fusion de régions différentes contrairement cette fois à la volonté des élus (le cas de la région de Vaudreuil sous influence urbaine, d'une part, et de celle de Soulanges tournée vers la campagne, d'autre part, qui furent pourtant fusionnées pour former les MRC de Vaudreuil-Soulanges). Parfois les unités paysagères, topographiques ou hydrographiques ont servi en tout ou en partie de référent à la délimitation de périmètres, bien que cette logique n'ait pas toujours été respectée en intégralité. À cet égard, le découpage de la MRC La Vallée-de-la-Gatineau illustre bien la logique de bassin versant (de la rivière Gatineau) qui a présidé sa délimitation. Cependant, la physionomie allongée de cette MRC fait en sorte que les problématiques des municipalités du sud diffèrent largement de celles du nord. Les premières (par exemple Low et Denholm) sont tournées vers l'agglomération Gatineau-Ottawa (en matière de flux de population), alors les dernières (par exemple Grand-Remous et Maniwaki) ont des préoccupations typiquement rurales (économique, forestière et récréotouristique). Autant dire que les frontières entre chacune des MRC correspondent en fait à un ensemble de critères variables selon les conditions administrative, géographique, historique, politique et sociale.
La délimitation de leur périmètre ne s'est pas faite sans heurts, et cet exercice répondait à des logiques différentes selon le contexte local et régional du moment.
L'histoire du développement régional au Québec retiendra que les périmètres des MRC circonscrivent des régions d'appartenance. Au moment de délimiter les MRC, et ce d'autant plus aujourd'hui, l'expression " région d'appartenance » demeure sujette à de multiples interprétations contradictoires compte tenu du sens mouvant des concepts de " région » et d' " appartenance » et des évolutions sociétales. Par exemple, des citoyens peuvent développer un sentiment d'appartenance à une région tout en travaillant et en consommant produits et services à l'extérieur de leur région d'appartenance. Dans ce cas de figure, comment traiter une problématique territoriale de développement socio-économique?

D'un point de vue théorique, la thèse s'inspire de Ascher ${ }^{7}$ selon lequel le processus de « modernisation des sociétés s'appuie sur une nouvelle forme du capi- 
talisme et sur l'économie cognitive ». Il donne naissance à une société dans laquelle "les individus ("pluriels" et "multi-appartenants") participent à des champs sociaux (le travail, la famille, le quartier, etc.) de plus en plus distincts et dans lesquels ils agissent de façons différenciées »). Cette thèse révise la notion d'appartenance pour plutôt insister sur de nouvelles priorités du développement dont celle, par exemple, qui consiste à produire de l'appartenance et de l'identité, donc des territoires différenciés.

\section{Contrairement à la politique des Pactes ruraux, l'État français ne s'est pas « enfermé » dans le découpage politico-administratif traditionnel en place lors de la mise en œuvre de la politique des Pays.}

\section{Des approches contractuelles voisines : Pacte, Pays et Parc naturel régional}

Pendant que Jean-Pierre Jolivet (ministre péquiste des Régions de 1998 à 2001) tentait d'accoucher de la première Politique nationale de la ruralité et du Pacte rural que le monde rural attendait avec impatience, la France mettait en branle et expérimentait une politique contractuelle similaire fondée sur les périmètres de « pays », elle-même inspirée des Parcs naturels régionaux.

\section{Politique nationale de la ruralité et Pays}

En France, une première Loi d'orientation pour le développement et l'aménagement $d u$ territoire (LOADT, dite loi Pasqua) en 1995 reconnaissait et instituait les territoires de Pays. La Loi d'orientation pour l'aménagement et le développement durable du territoire de 1999 (LOADDT, dite loi Voynet) reprend la question des Pays en lui donnant une place importante dans la politique d'aménagement du territoire.

Voynet fait de cet échelon territorial à la fois l'expression d'un projet associant la ville et la campagne; le lieu de participation et d'alliance entre les élus et les acteurs de la société civile; et l'échelon de contractualisation de l'État et des régions dans le cadre des contrats de plan État-Région. En faisant du Pays une sorte de fédération de communauté de communes à laquelle sont associés les autres acteurs économiques, sociaux, culturels et associatifs réunis au sein d'un conseil de développement, la LOADDT redonne du sens à la notion de Pays.

Un pays est un territoire caractérisé par une cohésion géographique, culturelle, économique et sociale au sein duquel :

- les collectivités territoriales et leurs groupements s'associent pour élaborer et mettre en œuvre une charte de développement durable, révisable dans les délais de dix ans, en coopération avec les représentants des activités économiques, sociales, culturelles et associatives réunis au sein d'un conseil de développement;

- l'État coordonne ses initiatives avec celles des collectivités locales dans le cadre d'un partenariat de contractualisation et tient compte de son périmètre pour l'organisation des services publics;

- son périmètre généralement plus large que celui des intercommunalités n'est pas tenu de respecter les limites administratives, mais doit en revanche respecter les périmètres des Établissements publics de coopération intercommunale (EPCI) à fiscalité propre;

- l'initiative de la création d'un Pays relève des communes et de leurs groupements, mais sa reconnaissance passe par l'élaboration de la charte qui permet d'associer les partenaires socioprofessionnels et associatifs en renforçant un partenariat entre élus et non élus.

Contrairement à la politique des Pactes ruraux, l'État français ne s'est pas "enfermé » dans le découpage politico-administratif traditionnel en place lors de la mise en œuvre de la politique des Pays. La Délégation à l'aménagement du territoire et à l'action régionale (DATAR) a plutôt questionné la bonne échelle d'intervention pour mieux laisser les acteurs locaux et les collectivités locales appréhender eux-mêmes les phénomènes socio-économiques et spatiaux au plus près de leurs réalités. À cette période de la politique des Pays, la DATAR et l'Institut national de la statistique et des études économiques ont identifié 348 «bassins d'emplois » correspondant chacun à une échelle théorique d'application du futur Pays. Selon eux, ce niveau «correspond à un cadre plus propice pour le 
développement économique et la mobilisation collective autour de projets territoriaux $\gg$.

Cette cartographie des mailles politico-administratives fut donc renouvelée au regard de nouveaux impératifs socio-économiques et de la mobilité des individus, alors que le Pacte rural évite pour le moment cette question fondamentale en se limitant au seul critère de la « cohésion » du découpage politicoadministratif en laissant de côté les critères de cohésion culturelle, économique et géographique.

\section{Pacte rural et Parc naturel régional}

La politique française des Pays (issue de la LOADT de 1995 et de la LOADDT de 1999) a suscité de vives réactions négatives chez les Parcs naturels régionaux. La légitimité de ces Parcs fut remise en question, car ils ont servis de modèle pour élaborer les Pays (l'idée de charte a été reprise tout comme les mandats dorénavant similaires) ${ }^{8}$.

Les Parcs naturels régionaux ont été créés en 1967 (il y en a aujourd'hui 45) à la suite d'une vaste réflexion qui connut son apogée au colloque de Lurs-enProvence de 1966 qui regroupa des fonctionnaires et des personnalités (ministres, architectes, aménageurs, présidents d'associations, artistes, etc.). Leurs débats reposaient sur plusieurs constats. Les années 1960 voient le développement rapide des zones urbaines, conséquence de la montée en puissance du «babyboom » et ce, au détriment du monde rural. La reconversion de vieilles régions industrielles comme le Nord-Pas-de-Calais était également d'actualité. Afin de changer l'image de la région en cause, l'idée est de requalifier le paysage régional afin d'attirer des services et des industries plus « modernes » que le charbon et séduire d'éventuels investisseurs étrangers. C'est cet argument qui a conduit à la création du tout premier Parc naturel régional, le Parc de Saint-AmandRaismes, dans le Nord. Enfin, la DATAR recherchait alors un outil institutionnel qui permette de préserver la nature de manière moins coûteuse et plus facile à mettre en place que le système (juridiquement contraignant) des Parcs nationaux, tout en y animant les secteurs ruraux (donc habités) en difficulté.

Les Parcs naturels régionaux sont créés à l'initiative de la Région pour des territoires ruraux dont le patrimoine naturel et culturel est riche, mais dont l'activité économique est souvent fragile. Ils permettent d'envi- sager le développement local de manière globale et durable, et remplissent pour cela cinq missions :

- protéger et mettre en valeur les richesses du patrimoine naturel et culturel, notamment par une gestion adaptée des milieux naturels et des paysages;

- contribuer à l'aménagement du territoire;

- contribuer à un développement économique, social, culturel et à la qualité de vie;

- assurer l'accueil, l'éducation et l'information du public;

- réaliser des actions expérimentales ou exemplaires dans les domaines cités ci-dessus et contribuer à des programmes de recherche ${ }^{9}$.

\section{Les Parcs naturels régionaux sont créés à l'initiative de la Région pour des territoires ruraux dont le patrimoine naturel et culturel est riche, mais dont I'activité économique est souvent fragile.}

Élaborée à partir d'un diagnostic du territoire concerné par le Parc, la Charte constitutive aux fondements pratiques et conceptuels du périmètre comporte :

- le projet de protection et de développement de ce territoire pour les douze ans à venir et les règles du jeu que se donnent les partenaires pour sa mise en œuvre;

- le plan du Parc qui décline les interventions prévues en fonction des particularités du territoire;

- les statuts et la composition de l'organisme de gestion du Parc, ses moyens financiers et humains;

- les instances consultatives (comités scientifiques, commissions et groupes de travail) de l'organisme de gestion;

- un programme d'actions prévisionnel à trois ans précis et chiffrés;

- le projet de convention d'application de la charte par l'État, convention signée par le Préfet de Région concerné, dès la création du Parc.

La politique des Parcs naturels régionaux est initiée, négociée, voulue et mise en œuvre par les élus locaux et adoptés par l'État lors du classement en Parc natu- 
rel régional, par décret du premier ministre. En signant volontairement la Charte du Parc, les Communes se fixent une règle du jeu commune et témoignent d'une volonté d'exigence vis-à-vis d'eux-mêmes selon les principes d'un accord moral et non contraignant. Les autres partenaires (non élus locaux) d'un Parc naturel régional sont les forces vives locales, c'est-à-dire les représentants socioprofessionnels (à travers leurs chambres consulaires, les syndicats professionnels, etc.) et les diverses associations (par exemple socioculturelles et d'usagers). Ils participent à l'élaboration de la Charte du Parc naturel régional en plus d'être associés à son fonctionnement et à ses programmes d'actions. Ils sont représentés, à titre consultatif, dans les commissions de travail et les instances du Parc, et sont les relais du Parc pour mener ses actions sur le terrain, dans le cadre de conventions spécifiques.

La charte est élaborée par un organisme d'étude (syndicat mixte, association, etc.) qui regroupe les collectivités concernées et leurs groupements et associe les organismes socioprofessionnels et associations locales. À l'expiration du classement après douze ans, la Charte constitutive doit être révisée par le Parc et présentée par la Région au ministère de l'Environnement compétent pour proposer un nouveau décret de classement $^{10}$. En cas de non-respect de la Charte du Parc, le ministre chargé de l'Environnement peut mettre fin au classement. L'organisme d'animation et de gestion du Parc est chargé de mettre en œuvre la charte, de veiller à son respect et d'assurer la cohérence des actions de ses partenaires sur le territoire du Parc. Son périmètre n'est soumis à aucune limite administrative, mais doit répondre à des critères de cohérence et de qualité en matière de patrimoine naturel, culturel et paysager. Au regard de ces critères, une commune peut adhérer au Parc pour une partie seulement de son territoire.

\section{Éloge de l'hypothèse territoriale faible}

L'arrivée du Pacte rural en 2001 s'est inscrite dans une logique administrative du développement et de la gestion de l'espace rural, tout en se calant sur le découpage politico-administratif en place. L'hypothèse territoriale retenue fut la plus faible et elle se traduit de la façon suivante: "Le Québec compte 86 MRC et autant de CLD sous leur responsabilité qui travaillent à la même échelle pour orchestrer le développement spatial et économique, alors instituons des Pac- tes ruraux à l'échelle des MRC tout en soustrayant celles qui ne sont pas éligibles $»^{11}$.

Cette hypothèse territoriale faible émane du document fondateur de la PNR II où on y apprend que « le territoire d'application de la Politique couvre 1011 municipalités et 34 communautés autochtones $\gg{ }^{12}$. On y apprend aussi que les municipalités d'Amos, Lachute, La Tuque, Cowansville, Matane et Dolbeau-Mistassini ont été intégrées au «territoire d'application de la politique » et ce, selon l'argumentaire que « ces municipalités présentent des caractéristiques similaires à des villes incluses dans un pacte rural, telles Montmagny, Sainte-Marie, Mont-Laurier ».

\section{L'arrivée du Pacte rural en 2001 s'est inscrite dans une logique administrative du développement et de la gestion de l'espace rural, tout en se calant sur le dé- coupage politico-administratif en place.}

Si la PNR II défendait réellement une approche territoriale (qui insiste, elle, sur la diversité des contextes culturel, économique, géographique et donc des problématiques), cet amalgame entre des municipalités aux profils très différents aurait été évité. Les auteurs du document justifient aussi leur décision en précisant que ces nouvelles municipalités incluses au «territoire d'application » de la PNR II « ont une aire d'influence qui se limite au territoire de leur MRC» et que leur «population urbaine est inférieure à 13000 habitants ». Cet argumentaire témoigne d'une conception limitée de l'approche territoriale car, dans les faits, comment cartographier l'aire d'influence d'une ville alors qu'elles évoluent toutes dans une économie mondialisée ? En vertu de quels critères le seuil des 13000 habitants a-t-il été retenu?

\section{Pacte rural 2014 : vers une triple approche Pays, Parc naturel régional et Municipalité régionale de comté ?}

Ce passage abordant la question de l'aire d'influence d'une ville ouvre une perspective intéressante que le gouvernement a évacuée (alors que, pourtant, l'essence de l'hypothèse territoriale s'y trouve). Les rapports verticaux (par exemple les rapports entre l'État et les collectivités locales) sont inextricablement liés aux rapports horizontaux (par exemple les rapports entre 
les flux de biens, de services et d'individus et l'espace du quotidien). Les pôles qui structurent des aires d'influence (par exemple. des bassins de vie) et des problématiques de développement rural (par exemple le manque d'appartenance) ne s'arrêtent pas au périmètre des MRC, d'où l'intérêt d'obliger les porteurs de Pacte rural à démontrer la pertinence du périmètre retenu au regard de leur réalité.

\section{Le nombre de Pactes ruraux devrait ainsi être limité, bien que le « pavage » à l'échelle du Québec puisse être complet.}

Il serait possible d'affirmer que le Pacte rural doit aussi être un outil :

1. à la disposition paritaire des citoyens, du public, des élus et des usagers d'un territoire;

2. autonome tant dans sa gestion, son fonctionnement que son financement;

3. dont le mandat est aussi de sensibiliser les décideurs (par exemple les élus), d'éduquer la population, de démontrer qu'il est possible par des actions concrètes d'allier des objectifs de développement aux objectifs de mise en valeur du patrimoine naturel et culturel;

4. qui contribue à produire de nouvelles connaissances en faisant lui-même (ou en faisant faire) des enquêtes et des analyses de son territoire (par exemple sur la faune, la flore et le patrimoine);

5. dont le périmètre est négocié, justifié et argumenté pour et par la population et les élus tant de «l'intérieur » que de «l'extérieur »;

6. que tous ne le méritent pas, ce qui signifie que le Québec rural ne serait pas nécessairement pavé de Pactes ruraux, comme c'est le cas aujourd'hui. Les porteurs de projet devraient démontrer que leur territoire (riche, mais fragile) est en mesure de tirer profit de la démarche et de l'outil Pacte rural. Cette démonstration devrait être refaite au terme du contrat afin de pouvoir renouveler la démarche, ce qui aurait pour avantage d'apporter de nouvelles idées, de réévaluer le profil socioéconomique du territoire et de remobiliser les acteurs autour d'un nouveau projet porteur.
Le nombre de Pactes ruraux devrait ainsi être limité, bien que le «pavage » à l'échelle du Québec puisse être complet. À l'heure actuelle, de nombreuses MRC pourtant voisines mènent les mêmes actions et ce, pour les mêmes raisons, alors qu'elles œuvrent dans deux Pactes ruraux différents. La démarche du Pacte rural pourrait être une occasion de mettre à l'avantplan une véritable réflexion sur le territoire rural (identité, spécificité des milieux, qualité de vie, difficultés socio-économiques, etc.) avant de passer nécessairement par le maillage politico-administratif.

L'organisme de gestion d'une démarche Pacte rural pourrait comporter différentes commissions (agriculture, environnement, paysage, économie, recherche, etc.) formées de représentants des élus, des citoyens et des différents acteurs locaux et régionaux). Si le Pacte rural entérine de nouveaux rapports contractuels intéressants entre l'État et les MRC, avec une clause « modulatoire verticale », l'autre génération de Pacte rural à venir devrait explicitement prévoir une clause de « modulation horizontale » afin de faciliter l'appréhension des problématiques territoriales par les acteurs de terrain.

\section{Notes et références}

1 Joyal, A. et El Batal, K. (2007). « Décentralisation et développement rural: étude de cas québécois ", Revue d'économie régionale et urbaine, $\mathrm{n}^{\mathrm{O}} 2$, p. 251-267.

2 SRQ (1999). Avis pour une politique gouvernementale de développement rural, Nicolet, Solidarité rurale du Québec, $31 \mathrm{p}$.

3 Allie, L. (2006). « Des parcs naturels habités au Québec? Une comparaison inspirée des Parcs naturels régionaux français », dans M. Simard et al. (dir.), Inégalités, démocratie et développement. Des enjeux pour la gouvernance des territoires locaux et régionaux, Rimouski, GRIDEQCRDT-ARUC-ÉS-GRIR, collection Tendances et débats en développement régional, $\mathrm{n}^{\circ} 11$, p. 303-320.

4 Gorgeu, Y et al. (1997). La charte de territoire, une démarche pour un projet de développement durable, Mairieconseils, Caisse des dépôts et Fédération des parcs naturels régionaux, Paris, La Documentation française, 311 p.

5 Proulx, M.-U. (1992). «Appartenances, utilités et fonctions. Le cas des petites régions MRC du Québec », Revue canadienne des sciences régionales, vol. XV, p. 307-325.

6 Fortin et Parent (1983). Les MRC et leur capacité d'extension, INRS-Urbanisation, Études et documents, $\mathrm{n}^{\circ} 38$, $86 \mathrm{p}$. 
7 Ascher, F. (2001). Ces événements nous dépassent, feignons d'en être les organisateurs, La Tour d'Aigues, Éditions de l'aube.

8 FPNRF (1997). Manifeste pour l'avenir des Parcs naturels régionaux, Paris, Gallimard.

9 Les Parcs naturels régionaux incarnent donc à la fois les objectifs du Pacte rural et des Laboratoires ruraux au sein d'un seul et même concept territorialisé de développement socio-économique. Cependant, la Pacte rural n'a pas de Charte constitutive ni d'organisme de gestion qui lui est entièrement dédié.
10 Le concept de Parc naturel régional est en fait une marque déposée à l'Institut national de la propriété intellectuelle dont le propriétaire est le ministère chargé de l'Environnement.

11 En définitive, le «territoire d'application » de la PNR II rejoint 86 MRC dont 75 sont entièrement couvertes et 21 partiellement.

12 Gouvernement du Québec (2006). Politique nationale de la ruralité. Une force pour tout le Québec, Ministère des Affaires municipales et des Régions, p. 23. 